\title{
A Folate- and Vitamin B12-Deficient Diet Decreases the Latency and Increases the Incidence of Mammary Tumors in MMTV-ErbB2 Transgenic Mice
}

\section{Zhengzheng Xiao ( $\nabla$ xiaozheng123@haust.edu.cn )}

The First Affiliated Hospital and College of Clinical Medicine of Henan University of Science and Technology

\section{Guoliang Yao}

The First Affiliated Hospital and College of Clinical Medicine of Henan University of Science and Technology

\section{Yongxuan Liu}

The First Affiliated Hospital and College of Clinical Medicine of Henan University of Science and Technology

\section{Chunling Zhao}

The First Affiliated Hospital and College of Clinical Medicine of Henan University of Science and Technology

\section{Research Article}

Keywords: breast cancer, hyperhomocysteinemia, vitamin B12, folate, metabolism

Posted Date: April 23rd, 2021

DOl: https://doi.org/10.21203/rs.3.rs-439338/v1

License: (c) (i) This work is licensed under a Creative Commons Attribution 4.0 International License. Read Full License 


\section{Abstract}

There has been controversy regarding folate- and vitamin B12-deficient diet (FVD)-induced hyperhomocysteinemia (HHcy) associated with breast cancer risk in most published epidemiological studies. Thus, the present study designed experiments to assess the causal association between FVDinduced HHcy and mammary tumor risk, as well as to identify the relative underlying mechanism. In this study, mammary tumor development was examined in mouse mammary tumor virus (MMTV)-erb-b2 receptor tyrosine kinase 2 (ErbB2) mice fed with a control AIN-93G diet or a FVD diet. MMTV-ErbB2 mice fed with the FVD diet displayed elevated blood levels of the amino acid homocysteine, a shorter tumor latency and an increased tumor multiplicity compared with the controls. The expression levels of key markers in the receptor tyrosine kinase and estrogen receptor (ER) signaling pathways, including phosphorylated (p)-Akt, p-Erk, p-ERa and Cyclin D1, were elevated in mammary tissues from MMTV-ErbB2 mice fed the FVD diet compared with mice fed with control diet. These data suggested that FVD-induced $\mathrm{HH} c y$ may promote mammary tumor development and decrease tumor latency, possibly by activating the epidermal growth factor receptor/ErbB2 and ERa signaling pathways. Therefore, examining the signaling mechanisms and identifying the relative metabolic pathways underlying mammary tumor promotion following FVD-induced HHcy may provide a novel strategy for breast cancer prevention and treatment.

\section{Introduction}

Breast cancer is the most common malignancy in women worldwide. In China, breast cancer remains the leading cause of female cancer-related mortality ${ }^{[1-3]}$. ErbB2 is amplified and pathologically upregulated in $25-30 \%$ of breast cancer cases, and is associated with poor prognosis ${ }^{[4]}$. Thus, ErbB2-positive breast cancer is a significant public health concern.

Hyperhomocysteinemia (HHcy), which is a term used to describe elevated blood levels of the amino acid homocysteine (Hcy), usually indicates a deficiency of vitamin B-12 and folate in the diet ${ }^{[5]}$. HHcy is observed in $\sim 5 \%$ of the general population ${ }^{[6,7]}$, and is associated with an increased risk for numerous disorders, including diabetes, renal disease, osteoporosis and different types of cancer ${ }^{[8-20]}$. Recently, accumulating evidence has identified higher prevalence of $\mathrm{HHcy}$ in breast cancer patients ${ }^{[7,21,22]}$. Moreover, $\mathrm{HH} c y$ has been shown to be associated with tumor suppressor epigenetic modulation in breast cancer patients $^{[21]}$. Based on these results, it was hypothesized that folate- and vitamin B12-deficient (FVD) diet-induced HHcy (FVD-induced HHcy) may increase breast cancer risk. At present, the relationship between FVD-induced HHcy and breast cancer remains controversial ${ }^{[7,21-23]}$. For instance, whether elevated Hcy levels are pathological consequences or a cause of breast cancer has not yet been clarified. This dilemma prompted us to examine the influence of FVD-induced HHcy on breast cancer initiation. Furthermore, understanding the mechanism responsible for the association between FVD-induced HHcy and breast cancer development may provide practical means to prevent or reduce the risk of breast cancer initiation. 
Over the past decade, the mouse mammary tumor virus (MMTV)-erb-b2 receptor tyrosine kinase 2 (ErbB2)-overexpressing transgenic breast cancer mouse model has been well-established ${ }^{[24-28]}$. MMTVErbB2 mice are ideal for testing the impact of various factors on ErbB2-induced mammary tumorigenesis ${ }^{[27-29]}$, as these mice can spontaneously develop nodular mammary tumors at $\sim 25$ weeks of age ${ }^{[27,28]}$. The long tumor latency of this animal model has significant advantages for mechanistic studies involving breast cancer etiology. Besides its use in genetically engineered models for breast cancer, FVD has long been used to induce moderate HHcy in mice and establish a HHcy mouse model ${ }^{[12]}$. As FVD-induced HHcy is observed in MMTV-ErbB2 mice, the present study examined whether FVDinduced HHcy affects mammary tumor development in MMTV-ErbB2 mice. The underlying mechanisms were also examined in the mammary tissues prior to malignant transformation. It was found that FVD induced moderate HHcy and promoted ErbB2-mediated carcinogenesis via the enhanced activation of epidermal growth factor receptor (EGFR) and estrogen receptor (ER) signaling. The present data could provide novel insights into the impact of FVD-induced HHcy in promoting breast cancer initiation, and it was suggested that a cancer-protective diet that is rich in folate, vitamin B6, and other nutrients may reduce breast cancer risk.

\section{Materials And Methods}

\section{Reagents and antibodies.}

As described in our previous study [28, 30-32], ErbB2 (cat. no. ab2165), phosphorylated (p)-ErbB2 (cat. no. Aab2241), estrogen receptor (ER)a (E115; cat. no ab32063) and Ki67 (cat. no. ab16667) primary antibodies were purchased from Abcam. Akt (cat. no. 4691), p-ERa (Ser118; cat. no. 2511) and p-Akt (Ser473; cat. no. 4060) primary antibodies were purchased from Cell Signaling Technology. Inc. cyclin D1 (cat. no. sc-8396), p-ERK (cat. no. sc-7383), Erk2 (cat. no. sc-1647), and $\beta$-actin (cat. no. sc-81178) primary antibodies were obtained from Santa Cruz Biotechnology, Inc. goat anti-rabbit IgG (cat. no. G21234) and Horseradish peroxidase (HRP)-conjugated goat anti-mouse lgG (cat. no. G-21040) secondary antibodies were obtained from Thermo Fisher Scientific, Inc.

\section{Animal treatment and sample collection.}

FVB/N-Tg (MMTV-ErbB2) transgenic mice (6 weeks of age,weight, 15-20 g) were obtained from Jackson Laboratory (Bar Harbor, ME, USA). Mice were housed in an environment with an ambient temperature of $22 \pm 2^{\circ} \mathrm{C}$, relative humidity of $50 \pm 10 \%$ and a $12 \mathrm{~h}$ light/dark cycle with food and water available ad libitum in the institutional animal facility. Animal care and experiments were approved by the Institutional Animal Care and Use Committee of Henan University of Science and Technology. All procedures were conducted in accordance with the National Institutes of Health Guide for the Care and Use of Laboratory Animals, 8th edition, 2011. The study was carried out in compliance with the ARRIVE guidelines. An AIN93G diet (LAD3001 diet; Trophic, NanJing, China) and a folate $(<0.2 \mathrm{mg} / \mathrm{kg})$ - and vitamin B12 $(<0.001$ $\mathrm{mg} / \mathrm{kg}$ )-deficient diet (FVD diet; Trophic, NanJing, China), identical to the AIN-93G diet except for the depletion of folate and vitamin B12, were used in the following experimental diets. 
A total of 26 female MMTV-ErbB2 mice were randomized at 6 weeks of age and placed into control group and FVD diet group ( $n=13$ mice per group). Beginning at 6 weeks of age, 13 MMTV-ErbB2 transgenic mice in the control group were fed the AIN-93G diet. The other 13 MMTV-ErbB2 transgenic mice in the FVD group were fed the FVD diet. After 10 weeks of FVD diet administration (at 16 weeks of age), three mice from each group were euthanized using $\mathrm{CO}_{2}$, with a $\mathrm{CO}_{2}$ fill rate of $30 \%$ of the chamber volume per minutes for 5 minutes, followed by cervical dislocation. Next, mammary tissues from mice at 16 weeks of age were collected for mammary gland whole mount, FACS and protein and DNA/RNA analyses.

As described in our previous study ${ }^{[27,28]}$, The remaining mice in MMTV-ErbB2 control and FVD group were monitored for tumor development with a twice-a-week examination beginning at 16 weeks of age. The dates of the first palpable mammary tumors, while was defined as the latency of tumor development, in each mouse were recorded. Mammary tumor development was observed twice a week from 18 weeks of age for all female mice. The end point of the experiments was defined as when the tumors reached 1.5 $\mathrm{cm}$ in diameter or mice had $>20 \%$ weight loss. tumor sizes were measured with calipers twice a week after palpable tumors were detected. Tumor latency and the percentages of tumor-free mice were also calculated. At the endpoint, mice were euthanized using $\mathrm{CO}_{2}$, with a $\mathrm{CO}_{2}$ fill rate of $30 \%$ of the chamber volume per minutes for 5 minutes, followed by cervical dislocation. Upon sacrifice, breast tumor tissue and mammary tissue were harvested for molecular and histological analyses.

Tumor volumes were calculated based on the formula: Tumor volume $=$ longest diameter $\times$ shortest diameter ${ }^{2} \times 0.5$. The tumor-free interval was presented with a Kaplan-Meier survival curve. Food intake was measured by weighing uneaten pellets. Tumor latency and tumor multiplicity were calculated at the endpoint of the experiment (when all mice had developed tumors).

\section{Hematoxylin and eosin staining.}

For histopathological analysis, the collected tissues were fixed overnight ( $18 \mathrm{~h}$ ) in 10\% neutral formalin at room temperature and paraffin-embedded. Mammary or tumor tissue sections ( $5 \mu \mathrm{m}$ thickness) were deparaffinized, and the slide was heated on a burner and placed in xylene. The tissue section was hydrated by passing through decreasing concentrations $(100 \%, 90 \%, 80 \%, 70 \%)$ of alcohol baths and water. Sections were stained at room temperature in hematoxylin for 3-5 minutes, and washed under running tap water until sections were "blue" for 5 minutes or less. Sections were differentiated in $1 \%$ acid alcohol ( $1 \% \mathrm{HCl}$ in $70 \%$ alcohol) for 5 minutes, and then washed under running tap water until the sections were blue again by dipping in an alkaline solution (ammonia water). Tissues were washed again with tap water and stained in $1 \%$ eosin $\mathrm{Y}$ at room temperature for 10 minutes. After washing in tap water for 1-5 minutes, sections were dehydrated in increasing concentrations of alcohol and cleared in xylene. Sections were mounted in mounting media and observed under a light Nikon ECLIPSE E100 microscope at a magnification of $\mathrm{x} 10, \mathrm{x} 40$ or $\mathrm{x} 100$.

\section{Mammary whole mount preparation.}


The mammary gland was dissected and placed on a glass slide. Then, the tissue was fixed with $\mathrm{O} / \mathrm{N}$ in Carnoy's fixative (6:3:1 ratio of 100\% ethanol:chloroform:glacial acetic acid) at room temperature overnight. The following day, the tissues were dehydrated and the glands were stained. After washing in $70 \% \mathrm{EtOH}$ twice for 15 minutes, $50 \%$ EtOH twice for 10 minutes, $30 \%$ EtOH twice for 10 minutes, 10\% $\mathrm{EtOH}$ twice for 10 minutes and distilled $\mathrm{H}_{2} \mathrm{O}$ once for 5 minutes, tissues were stained using $\mathrm{O} / \mathrm{N}$ in carmine alum stain (1 g carmine; cat. no. C1022; Sigma-Aldrich; Merck KGaA) and $2.5 \mathrm{~g}$ aluminum potassium sulfate (cat. no. A7167; Sigma-Aldrich; Merck KGaA) in $500 \mathrm{~mL} \mathrm{dH}_{2} \mathrm{O}$ and boiled for 20 minutes. The final volume was adjusted to $500 \mathrm{~mL}$ with $\mathrm{H}_{2} \mathrm{O}$. The tissues were filtered and thymol crystal was added as the preservative. The following day, the cleared and mounted glands washed in $70 \% \mathrm{EtOH}$ twice for 15 minutes, 95\% EtOH twice for 15 minutes, 100\% EtOH twice for 15 minutes and cleared in xylene for $\sim 30$ minutes (or until fat was sufficiently cleared from the glands). Sections were mounted with $\sim 1 \mathrm{~mL}$ SecureMount and placed on a glass cover slip. The ductal architecture of whole mounts was examined under a light Nikon Eclipse 80i microscope and images were captured using the Nikon Elements Imaging System (Nikon Instruments, Inc.).

\section{Immunohistochemical staining.}

As described in our previous study ${ }^{[33]}$, tumor specimens were derived from formalin-fixed, paraffinembedded tissue samples. Following antigen retrieval and blocking with $10 \%$ goat serum at room temperature for $1 \mathrm{~h}$, tumor tissues were incubated with Ki67 (diluted 1:100) antibody overnight at $4^{\circ} \mathrm{C}$. The slides were then incubated with biotinylated anti-rabbit IgG secondary antibody (cat. no. PK-7200; 1:1,000 dilution; Vectastain Elite ABC-HRP kit; Vector Laboratories, Inc.) for 30 minutes at room temperature, followed by incubation in Vectastain Elite ABC Reagent for 30 minutes at room temperature. For all slides, a diaminobenzidine detection kit (Vector Laboratories, Inc.) was used according to the manufacturer's protocol. The sections were then counterstained with Meyer's hematoxylin for 8 minutes at $37^{\circ} \mathrm{C}$, dehydrated and mounted. Slides were observed and imaged using a light Nikon ECLIPSE E100 microscope at a magnification of $x 40$ or $\times 100$.

\section{Real time quantitative PCR (RT-qPCR).}

For qPCR, total RNA was extracted using TRIzol® reagent (cat. no. 15596026; Thermo Fisher Scientific, Inc.), and $1 \mu \mathrm{g}$ RNA was used for the RT reaction with random primer (cat. no. 48190011; Thermo Fisher Scientific, Inc.), dNTP mix (cat. no.18427013; Thermo Fisher Scientific, Inc.) and M-MuLV Reverse Transcriptase (cat. no. M0253L; New England Biolabs). mRNA quantification was performed using a SYBRGreen supermix (cat. no. 1708880; Bio-Rad Laboratories, Inc.). The PCR conditions were as follows: Initial denaturation at $94^{\circ} \mathrm{C}$ for 2 min, followed by 40 cycles of $94^{\circ} \mathrm{C}$ for $30 \mathrm{sec}, 56^{\circ} \mathrm{C}$ for $30 \mathrm{sec}, 72^{\circ} \mathrm{C}$ for $30 \mathrm{sec}$ and $20-60 \mathrm{sec}$ at $72^{\circ} \mathrm{C}$. The triplicate samples were amplified in $20 \mu \mathrm{l}$ reactions with gene-specific primers. The mRNA abundance for each gene of interest was normalized to that of GAPDH. The PCR primers used were as follows: ERBB2 forward, 5'-CAGCCCCAGAGGATTACAGA-3' and reverse, 5'TCAGTCCTAGTGGGGTGTCC-3'; ERa forward, 5'-TCTCTGGAAGAGAAGGACCACATC-3' and reverse, 5'TGCAGAGTCAGGCCAGCTTT-3'; Cyclin D1 forward, 5'-GGGCACCTGGATTGTTCT-3' and reverse, 5'- 
CACCGGAGACTCAGAGCA-3'; GAPDH forward, 5'-GAACATCATCCCTGCCTCTACT-3' and reverse, 5'CGCCTGCTTCACCACCTT-3'.

\section{Western blot analysis.}

The protocol was described in our previous study ${ }^{[33]}$, equal amounts of protein (30 $\left.\mu \mathrm{g}\right)$ were separated by $10 \%$ SDS-PAGE, and transferred onto nitrocellulose membranes. After blocking in $5 \%$ milk in TBST, the membranes were incubated in diluted primary antibodies $(1: 1,000)$ overnight at $4^{\circ} \mathrm{C}$. Then the membranes were washed and incubated in horseradish peroxidase (HRP) labeled secondary antibodies $(1: 3,000)$ for 1.5 hours at room temperature. SuperSignal West Pico ECL solution (Thermo Fisher Scientific) was added to the membranes to enhance the chemiluminescent signal. Proteins bands were imaged using a FluorChemE imager.

\section{Determination of the plasma Hcy levels.}

The total plasma Hcy levels were measured using a Hcy ELISA Kit (cat. no. EU0197l; Fine Biotech Co., Ltd.) according to manufacturer's instruction. The antibody was diluted to 1:1,000 in CBS and coated a 96-well microplate with $100 \mu \mathrm{L}$ per well of the diluted capture antibody. A total of $100 \mu \mathrm{L}$ sample or standards were added in sample dilution buffer per well, and the plate was sealed and incubated for $2 \mathrm{~h}$ at room temperature. Then, $100 \mu \mathrm{L}$ detection antibody was added to each well, and the plate was sealed and incubated for $1 \mathrm{~h}$ at room temperature. A total of $200 \mu \mathrm{L}$ substrate solution was added to each well and incubated for 20 minutes at room temperature. Then, $50 \mu \mathrm{L}$ stop solution was added to each well, and the plate was taped to ensure thorough mixing. The optical density of each well was determined immediately using a microplate reader set to $450 \mathrm{~nm}$. The concentration of the sample was measured using ELISA Analysis Software.

\section{Statistical analysis.}

Kaplan-Meier curves were generated and used to compare the tumor onset between the control and FVD diet groups. A log-rank (Mantel-Cox) test was used to determine whether the Kaplan-Meier curves were significantly different. A Fisher's Exact test was used to compare the number of mice that developed mammary tumors between the two diets. A Student's t-test was used when comparing gene expression between the two diets. Data are presented as the mean $\pm S D$. $P<0.05$ was considered to indicate a statistically significant difference.

\section{Results}

\section{Administration of FVD diet induces HHcy in MMTV-ErbB2 mice.}

It is known that a FVD diet effectively elevated homocysteine levels and resulted in HHcy in different mouse models. In the current study, a total of 26 MMTV-ErbB2 mice (6-week-old) were fed with a FVD diet (diet group) or Vehicle diet (control group) for 16 weeks. During the dietary intervention, no significant 
difference in the body-weight and daily intake between these two groups was found. The FVD dietary intervention increased the Hcy levels from below the limit of detection $(5 \mu \mathrm{mol} / \mathrm{L})$ to $14.3 \pm 0.8 \mu \mathrm{mol} / \mathrm{L}$ after 4 weeks. After 8 weeks of intervention, the Hcy levels of the diet group were significantly higher compared with those of the control group, and remained at $\sim 18.3 \pm 2.8 \mu \mathrm{mol} / \mathrm{L}$ (Fig. 1). At the end of the diet treatment, body weight and plasma total cholesterol and triglycerides levels were not significantly different between control and FVD groups. No weight loss, loss of appetite and fatigue were observed. These data demonstrated that administration of FVD diet for $>4$ weeks can significantly induced HHcy in MMTV-ErbB2 mice.

\section{FVD-induced HHcy promotes mammary tumorigenesis in MMTV-ErbB2 transgenic mice.}

Since HHcy was induced in MMTV-ErbB2 mice after 4 weeks of FVD dietary intervention, the effects of FVD-induced HHcy on ErbB2-mediated mammary tumorigenesis were examined. In the present study, the MMTV-ErbB2 mice were administered with FVD diet from week 6 to 22 (16 weeks). The tumor latency and the average number of palpable tumors per mouse was examined. In the FVD group, mice developed palpable tumors between 20 and 41 weeks of age with an average latency of 30 weeks. In the control group, mice developed palpable tumors between 28 and 52 weeks of age with an average latency of 39.7 weeks. The total number of tumors in control or FVD mice group was 11 and 18, respectively (Fig. 2). These results suggested that FVD induced HHcy decreased the latency and increased the incidence of mammary tumors in MMTV-ErbB2 Transgenic Mice.

\section{FVD-induced HHcy causes proliferative mammary morphogenesis in MMTV-ErbB2 mice.}

It is known that an increased breast cancer risk is associated with advanced mammary gland development and increased ductal and epithelial density ${ }^{[24-26]}$. In order to evaluate the effect of FVD induced HHcy on the premalignant mammary morphology in the MMTV-ErbB2 mice, the elongation of the mammary ductal and number of terminal end bud (TEB)-like structures were examined in the premalignant mammary gland. FVD-induced HHcy promoted mammary ductal elongation and increased the number of TEB-like structures in the 16-week-old MMTV-ErbB2 mice. Mammary glands from FVD mice also displayed increased nuclear staining of the proliferation marker BrdU (Fig. 3). These results indicated that FVD-induced HHcy promoted mammary gland developmental changes in premalignant mammary gland tissues.

\section{FVD-induced HHcy promotes ErbB2/mitogen-activated protein kinase (MAPK)/phosphatidylinositol 3- kinase (PI3K)/Akt signaling in MMTV-ErbB2 Mice.}

It has been reported that ErbB2/MAPK/PI3K/Akt signaling is the driving force of ErbB2-mediated carcinogenesis $^{[25,29,34-36]}$. Since mammary gland development was promoted in premalignant mammary gland tissues of MMTV-ErbB2 mice, the protein and mRNA expression levels of ErbB2, Akt, and Erk of the premalignant mammary tissues were examined. It was found that the protein and mRNA expression levels of ErbB2, Akt, and Erk were significantly increased by FVD dietary intervention (Fig. 4). 
These results suggested that ErbB2/MAPK/PI3K/Akt signaling may be involved in FVD dietary intervention-induced mammary gland developmental changes in MMTV-ErbB2 mice.

\section{FVD-induced HHcy promotes ER signaling in MMTV-ErbB2 Mice.}

Since HHcy has been reported to exert an estrogen-like effect ${ }^{[10-12,37,38]}$, the activation of ERa and its target genes were examined in premalignant mammary gland tissues of MMTV-ErbB2 mice. The protein and mRNA expression levels of ERa and Cyclin D1 in the premalignant mammary were investigated. The western blotting and RT-PCR results demonstrated that FVD dietary intervention significantly increased the protein and mRNA expression levels of ERa and Cyclin D1 (Fig. 4). Moreover, in the present preexperiment, six parental FVB/N mice were randomly assigned to fed with either control or FVD diet $(n=3$ mice per group). After 1 month, mammary gland tissues displayed elevated $\mathrm{p}$-ERa and $\mathrm{p}$-Akt protein expression levels (data not shown). These results suggested that ERa signaling was involved in the FVD dietary intervention-induced mammary gland developmental changes in MMTV-ErbB2 mice.

\section{Discussion}

Breast cancer is the most commonly occurring malignancy in women, impacting 2.1 million women each year, and also causes the greatest number of cancer-related mortalities among women ${ }^{[1,2,4]}$. Identifying possible risk factors is a vital part of breast cancer prevention. It has been reported that elevated plasma Hcy may induce oxidative damage and metabolic disorders, and subsequently lead to cancer carcinogenesis $[5,6,10,13,14,38-40]$. High Hcy levels may indicate deficiencies in vitamins B-12 and folate. The primary cause of FVD is low intake of sources rich in the vitamin, such as legumes, green leafy vegetables and animal-source foods, as well as malabsorption ${ }^{[7,18,39,41,42]}$. As folate and vitamin B12 have been reported to promote the conversion of Hcy to methionine by acting as a cofactor of the enzymes methionine synthase, deficiencies of vitamin $\mathrm{B} 12$ and folate can result in $\mathrm{HHcy}{ }^{[12]}$. Previous studies have revealed that the prevalence of subclinical FVD was $>10 \%$ and HHcy was $>30 \%$ in the Spanish vegetarian population ${ }^{[43]}$. Moreover, $>50 \%$ of vegetarian Indian subjects present with deficient levels of serum vitamin B12 and HHcy ${ }^{[21]}$. Epidemiological data regarding folate and vitamin B12 among the women in Shaanxi Provence, China also revealed that $\sim 14.7 \%$ of the women were deficient in folate and $45.5 \%$ of the women were deficient in vitamin $B 12^{[7,44]}$. It is estimated that $\geq 10 \%$ of the United States population has low folate stores, while the prevalence of vitamin B12 deficiency has been estimated to be $\sim 16 \%{ }^{[45]}$. These data indicate folate and B12 deficiencies represent public health problems worldwide. More worryingly, it has been documented that individuals with low serum folate and B12 levels and elevated serum Hcy levels are prone to various pathologies, including atherosclerosis, cardiovascular disease, birth defects, ischemic stroke, venous thromboembolism and cancer $[5,8,10-18,20-$ $22,37,38,43-51]$.

Investigations into the effects of FVD-induced HHcy on breast cancer risk have yielded conflicting results. For example, a prospective case-control study of 812 patients with invasive breast cancer and 812 
matched controls followed up for 10 years between 1992 and 2004 found that plasma levels of Hcy were not associated with overall breast cancer risk ${ }^{[52]}$. However, recent epidemiological and clinical studies examining China and Mexican populations found an increased incidence of HHcy and elevated plasma Hyc levels in patients with breast cancer ${ }^{[7,22]}$. Furthermore, Hcy levels were found to be positively associated with the proliferation rates of breast tumor ${ }^{[22]}$. Methylenetetrahydrofolate reductase haplotypes also significantly affect breast cancer occurrence ${ }^{[21]}$. These data suggest that HHcy in certain high-risk individuals may increase breast cancer risk.

Although numerous previous studies have reported positive associations between Hcy levels and breast cancer risk, it cannot be verified whether the elevated Hcy levels are pathological consequences or a cause of breast cancer. In order to clarify this issue, the present study induced HHcy using FVD dietary intervention in MMTV-ErbB2 overexpressing transgenic breast cancer mice, and examined the impact of FVD-induced HHcy on mammary tumorigenesis.

The ErbB2 gene belongs to the EGFR family of receptor tyrosine kinases ${ }^{[29,35,36]}$. Activation of ErbB2 receptor signaling pathways can promote various human malignancies including medulloblastoma, lung, prostate and breast cancer ${ }^{[53]}$. The ErbB2 gene is amplified and/or upregulated in approximately one-third of human breast carcinomas, and has been widely reported to promote breast tumorigenesis via the activation of multiple ErbB2 downstream signals, such as Shc/growth factor receptor-bound protein 2Ras,PI3K-Akt, MEK-ERK, JNK, p38 mitogen-activated protein kinase, protein kinase $\mathrm{C}$ and phospholipase $C$, amongst others ${ }^{[29,35,36,53]}$. MMTV long terminal repeat has most frequently been used to express a gene of interest in the mammary epithelium, and overexpression of ErbB2 via the MMTV promoter leads to the onset of multiple mammary tumors at a median age of 6-10 months in transgenic mice, with the frequent potential to metastasize to the lung ${ }^{[24-26,28]}$. Thus, MMTV-neu/ErbB2 transgenic mice have been created and are recognized as excellent models for breast cancer. In the present study, FVD-induced HHcy caused distinct mammary morphogenic changes and promoted mammary tumor development in MMTV-ErbB2 mice. To the best of our knowledge, these data provide the first direct in vivo evidence that FVD-induced HHcy can promote mammary tumorigenesis.

Despite the progress in confirming the role of FVD-induced HHcy in promoting mammary tumorigenesis, the molecular signaling mechanisms that are responsible for HHcy-mediated mammary tumorigenesis are largely unknown. It has been reported that activation of ErbB2/MAPK/PI3K/Akt signaling is the driving force of ErbB2-mediated carcinogenesis ${ }^{[29,35,36]}$. Moreover, previous reports have revealed that $\mathrm{HH}$ cy causes S-adenosylhomocysteine intracellular accumulation, and induces the oxidative metabolites of estrogens (catechol estrogens) ${ }^{[21]}$. Catechol estrogens further promote breast tumor formation via estrogen signaling ${ }^{[10,38,54,55]}$. Previous reports also suggested that high levels of catechol estrogens are associated with increased breast cancer risk ${ }^{[22,23]}$. Based on these data, the present study examined the effects of FVD-induced HHcy on activation of ErbB2/MAPK/PI3K/Akt and ERa signaling in premalignant mammary glands. The current data indicated that FVD-induced HHcy significantly activated ErbB2/MAPK/PI3K/Akt and ERa pathways. Since ErbB2-ER signaling crosstalk has widely reported to 
promote mammary tumor development $[27,56]$, it was suggested that ErbB2-ER signaling crosstalk may participate in FVD-induced HHcy mediated tumor development in MMTV-ErbB2 mice. These results suggested the ErbB2-ER signaling activation was due to FVD administration, and was not specific to MMTV-ErbB2 activation in MMTV-ErbB2 mice.

In conclusion, the present study demonstrated that a FVD diet induced HHcy in the MMTV-ErbB2 mouse breast cancer model, and that FVD-induced HHcy accelerated mammary tumor development. Since ErbB2/MAPK/PI3K/Akt and ERa signaling serves a central role in breast cancer pathogenesis, the present results further support the concept that dietary FVD was associated with HHcy, and may contribute to mammary tumorigenesis via the ErbB2/MAPK/PI3K/Akt and ERa signaling pathways.

\section{References}

[1] Fan L, Strasser-Weippl K, Li JJ, et al. Breast cancer in China. Lancet Oncol. 2014. 15(7): e279-89.

[2] Feng RM, Zong YN, Cao SM, Xu RH. Current cancer situation in China: good or bad news from the 2018 Global Cancer Statistics. Cancer Commun (Lond). 2019. 39(1): 22.

[3] Li Y, Li Q, Mo H, et al. Incidence, risk factors and survival of patients with brain metastases at initial metastatic breast cancer diagnosis in China. Breast. 2020. 55: 30-36.

[4] Tan YO, Han S, Lu YS, et al. The prevalence and assessment of ErbB2-positive breast cancer in Asia: a literature survey. Cancer. 2010. 116(23): 5348-57.

[5] Hasan T, Arora R, Bansal AK, Bhattacharya R, Sharma GS, Singh LR. Disturbed homocysteine metabolism is associated with cancer. Exp Mol Med. 2019. 51(2): 1-13.

[6] Chen S, Guo X, Dong S, Li Z, Sun Y. Relationship between lifestyle factors and hyperhomocysteinemia in general Chinese population: a cross-sectional study. Postgrad Med. 2017. 129(2): 216-223.

[7] Song A, Zhao L, Li Y, et al. Haplotypes of the MTHFR gene are associated with an increased risk of breast cancer in a Han Chinese population in Gansu province. IUBMB Life. 2016. 68(7): 526-34.

[8] Zhang D, Lou J, Zhang X, et al. Hyperhomocysteinemia results from and promotes hepatocellular carcinoma via CYP450 metabolism by CYP2J2 DNA methylation. Oncotarget. 2017. 8(9): 15377-15392.

[9] Braun DJ, Abner E, Bakshi V, et al. Blood Flow Deficits and Cerebrovascular Changes in a Dietary Model of Hyperhomocysteinemia. ASN Neuro. 2019. 11: 1759091419865788.

[10] Forges T, Monnier-Barbarino P, Alberto JM, Guéant-Rodriguez RM, Daval JL, Guéant JL. Impact of folate and homocysteine metabolism on human reproductive health. Hum Reprod Update. 2007. 13(3): 225-38. 
[11] McCaddon A. Vitamin B12 in neurology and ageing; clinical and genetic aspects. Biochimie. 2013. 95(5): 1066-76.

[12] Troen AM, Shea-Budgell M, Shukitt-Hale B, Smith DE, Selhub J, Rosenberg IH. B-vitamin deficiency causes hyperhomocysteinemia and vascular cognitive impairment in mice. Proc Natl Acad Sci U S A. 2008. 105(34): 12474-9.

[13] Giovannucci E. Epidemiologic studies of folate and colorectal neoplasia: a review. J Nutr. 2002. 132(8 Suppl): 2350S-2355S.

[14] Harnack L, Jacobs DR Jr, Nicodemus K, Lazovich D, Anderson K, Folsom AR. Relationship of folate, vitamin B-6, vitamin B-12, and methionine intake to incidence of colorectal cancers. Nutr Cancer. 2002. 43(2): $152-8$.

[15] Qin T, Du M, Du H, Shu Y, Wang M, Zhu L. Folic acid supplements and colorectal cancer risk: metaanalysis of randomized controlled trials. Sci Rep. 2015. 5: 12044.

[16] Passarelli MN, Barry EL, Zhang D, et al. Risk of basal cell carcinoma in a randomized clinical trial of aspirin and folic acid for the prevention of colorectal adenomas. Br J Dermatol. 2018. 179(2): 337-344.

[17] Chandy S, Sadananda Adiga MN, Ramaswamy G, Ramachandra C, Krishnamoorthy L. Effect of Vitamin B(12) and Folate on Homocysteine levels in colorectal cancer. Indian J Clin Biochem. 2008. 23(3): 258-61.

[18] Sugiyama A, Awaji H, Horie K, Kim M, Nakata R. The beneficial effect of folate-enriched egg on the folate and homocysteine levels in rats fed a folate- and choline-deficient diet. J Food Sci. 2012. 77(12): $\mathrm{H} 268-72$.

[19] Wei Z, Tiandong W, Yang L, et al. Parkinson's Disease and Homocysteine: A Community-Based Study in a Folate and Vitamin B12 Deficient Population. Parkinsons Dis. 2016. 2016: 9539836.

[20] Meng H, Li Y, Zhang W, Zhao Y, Niu X, Guo J. The relationship between cognitive impairment and homocysteine in a B12 and folate deficient population in China: A cross-sectional study. Medicine (Baltimore). 2019. 98(47): e17970.

[21] Naushad SM, Reddy CA, Kumaraswami K, et al. Impact of hyperhomocysteinemia on breast cancer initiation and progression: epigenetic perspective. Cell Biochem Biophys. 2014. 68(2): 397-406.

[22] Varela Almanza KM, Puebla-Pérez AM, Delgado-Saucedo Jl, et al. Increased homocysteine plasma levels in breast cancer patients of a Mexican population. Exp Oncol. 2018. 40(2): 114-118.

[23] Al-Awadi F, Yang M, Tan Y, Han Q, Li S, Hoffman RM. Human tumor growth in nude mice is associated with decreased plasma cysteine and homocysteine. Anticancer Res. 2008. 28(5A): 2541-4. 
[24] Ma Z, Parris AB, Howard EW, et al. Caloric restriction inhibits mammary tumorigenesis in MMTVErbB2 transgenic mice through the suppression of ER and ErbB2 pathways and inhibition of epithelial cell stemness in premalignant mammary tissues. Carcinogenesis. 2018. 39(10): 1264-1273.

[25] Zhao Q, Parris AB, Howard EW, et al. FGFR inhibitor, AZD4547, impedes the stemness of mammary epithelial cells in the premalignant tissues of MMTV-ErbB2 transgenic mice. Sci Rep. 2017. 7(1): 11306.

[26] Zhang GP, Han D, Liu G, et al. Effects of soy isoflavone and endogenous oestrogen on breast cancer in MMTV-erbB2 transgenic mice. J Int Med Res. 2012. 40(6): 2073-82.

[27] Ma Z, Kim YM, Howard EW, et al. DMBA promotes ErbB2-mediated carcinogenesis via ErbB2 and estrogen receptor pathway activation and genomic instability. Oncol Rep. 2018. 40(3): 1632-1640.

[28] Ma Z, Parris AB, Xiao Z, et al. Short-term early exposure to lapatinib confers lifelong protection from mammary tumor development in MMTV-erbB-2 transgenic mice. J Exp Clin Cancer Res. 2017. 36(1): 6.

[29] Slamon DJ, Clark GM, Wong SG, Levin WJ, Ullrich A, McGuire WL. Human breast cancer: correlation of relapse and survival with amplification of the HER-2/neu oncogene. Science. 1987. 235(4785): 177-82.

[30] Xiao Z, Yang X, Zhang K, et al. Estrogen receptor a/prolactin receptor bilateral crosstalk promotes bromocriptine resistance in prolactinomas. Int J Med Sci. 2020. 17(18): 3174-3189.

[31] Xiao Z, Liang J, Deng Q, et al. Pimozide augments bromocriptine lethality in prolactinoma cells and in a xenograft model via the STAT5/cyclin D1 and STAT5/Bcl-xL signaling pathways. Int J Mol Med. 2021. 47(1): 113-124.

[32] Xiao Z, Yang X, Liu Z, et al. GASC1 promotes glioma progression by enhancing NOTCH1 signaling. Mol Med Rep. 2021. 23(5).

[33] Ostrom QT, Cote DJ, Ascha M, Kruchko C, Barnholtz-Sloan JS. Adult Glioma Incidence and Survival by Race or Ethnicity in the United States From 2000 to 2014. JAMA Oncol. 2018. 4(9): 1254-1262.

[34] DiGiovanna MP, Stern DF, Edgerton SM, Whalen SG, Moore D 2nd, Thor AD. Relationship of epidermal growth factor receptor expression to ErbB-2 signaling activity and prognosis in breast cancer patients. J Clin Oncol. 2005. 23(6): 1152-60.

[35] Yarden Y, Sliwkowski MX. Untangling the ErbB signalling network. Nat Rev Mol Cell Biol. 2001. 2(2): 127-37.

[36] Freudenberg JA, Wang Q, Katsumata M, Drebin J, Nagatomo I, Greene MI. The role of HER2 in early breast cancer metastasis and the origins of resistance to HER2-targeted therapies. Exp Mol Pathol. 2009. 87(1): 1-11. 
[37] Ghemrawi R, Pooya S, Lorentz S, et al. Decreased vitamin B12 availability induces ER stress through impaired SIRT1-deacetylation of HSF1. Cell Death Dis. 2013. 4: e553.

[38] Pooya S, Blaise S, Moreno Garcia M, et al. Methyl donor deficiency impairs fatty acid oxidation through PGC-1a hypomethylation and decreased ER-a, ERR-a, and HNF-4a in the rat liver. J Hepatol. 2012. 57(2): 344-51.

[39] Burdge GC, Lillycrop KA. Nutrition, epigenetics, and developmental plasticity: implications for understanding human disease. Annu Rev Nutr. 2010. 30: 315-39.

[40] Vollset SE, Clarke R, Lewington S, et al. Effects of folic acid supplementation on overall and sitespecific cancer incidence during the randomised trials: meta-analyses of data on 50,000 individuals. Lancet. 2013. 381(9871): 1029-36.

[41] Mason JB. Folate, cancer risk, and the Greek god, Proteus: a tale of two chameleons. Nutr Rev. 2009. 67(4): 206-12.

[42] Guéant JL, Namour F, Guéant-Rodriguez RM, Daval JL. Folate and fetal programming: a play in epigenomics. Trends Endocrinol Metab. 2013. 24(6): 279-89.

[43] Gallego-Narbón A, Zapatera B, Barrios L, Vaquero MP. Vitamin B12 and folate status in Spanish lacto-ovo vegetarians and vegans. J Nutr Sci. 2019. 8: e7.

[44] Ronnenberg AG, Goldman MB, Aitken IW, Xu X. Anemia and deficiencies of folate and vitamin B-6 are common and vary with season in Chinese women of childbearing age. J Nutr. 2000. 130(11): 270310 .

[45] Guralnik JM, Eisenstaedt RS, Ferrucci L, Klein HG, Woodman RC. Prevalence of anemia in persons 65 years and older in the United States: evidence for a high rate of unexplained anemia. Blood. 2004. 104(8): 2263-8.

[46] Ninomiya T, Kiyohara Y, Kubo M, et al. Hyperhomocysteinemia and the development of chronic kidney disease in a general population: the Hisayama study. Am J Kidney Dis. 2004. 44(3): 437-45.

[47] Mohammad G, Kowluru RA. Homocysteine Disrupts Balance between MMP-9 and Its Tissue Inhibitor in Diabetic Retinopathy: The Role of DNA Methylation. Int J Mol Sci. 2020. 21(5).

[48] Chen S, Dong Z, Cheng M, et al. Homocysteine exaggerates microglia activation and neuroinflammation through microglia localized STAT3 overactivation following ischemic stroke. $J$ Neuroinflammation. 2017. 14(1): 187.

[49] Hanley MP, Kadaveru K, Perret C, Giardina C, Rosenberg DW. Dietary Methyl Donor Depletion Suppresses Intestinal Adenoma Development. Cancer Prev Res (Phila). 2016. 9(10): 812-820. 
[50] Figueiredo JC, Mott LA, Giovannucci E, et al. Folic acid and prevention of colorectal adenomas: a combined analysis of randomized clinical trials. Int J Cancer. 2011. 129(1): 192-203.

[51] Cole BF, Baron JA, Sandler RS, et al. Folic acid for the prevention of colorectal adenomas: a randomized clinical trial. JAMA. 2007. 297(21): 2351-9.

[52] Lin J, Lee IM, Song Y, et al. Plasma homocysteine and cysteine and risk of breast cancer in women. Cancer Res. 2010. 70(6): 2397-405.

[53] Hynes NE, Lane HA. ERBB receptors and cancer: the complexity of targeted inhibitors. Nat Rev Cancer. 2005. 5(5): 341-54.

[54] Song Q, Chen Y, Wang J, et al. ER stress-induced upregulation of NNMT contributes to alcoholrelated fatty liver development. J Hepatol. 2020. 73(4): 783-793.

[55] Ning H, Sun Z, Liu Y, et al. Insulin Protects Hepatic Lipotoxicity by Regulating ER Stress through the $\mathrm{PI3K} /$ Akt/p53 Involved Pathway Independently of Autophagy Inhibition. Nutrients. 2016. 8(4): 227.

[56] Ma Z, Parris AB, Howard EW, et al. In Utero Exposure to Bisphenol a Promotes Mammary Tumor Risk in MMTV-Erbb2 Transgenic Mice Through the Induction of ER-erbB2 Crosstalk. Int J Mol Sci. 2020. 21(9).

\section{Figures}



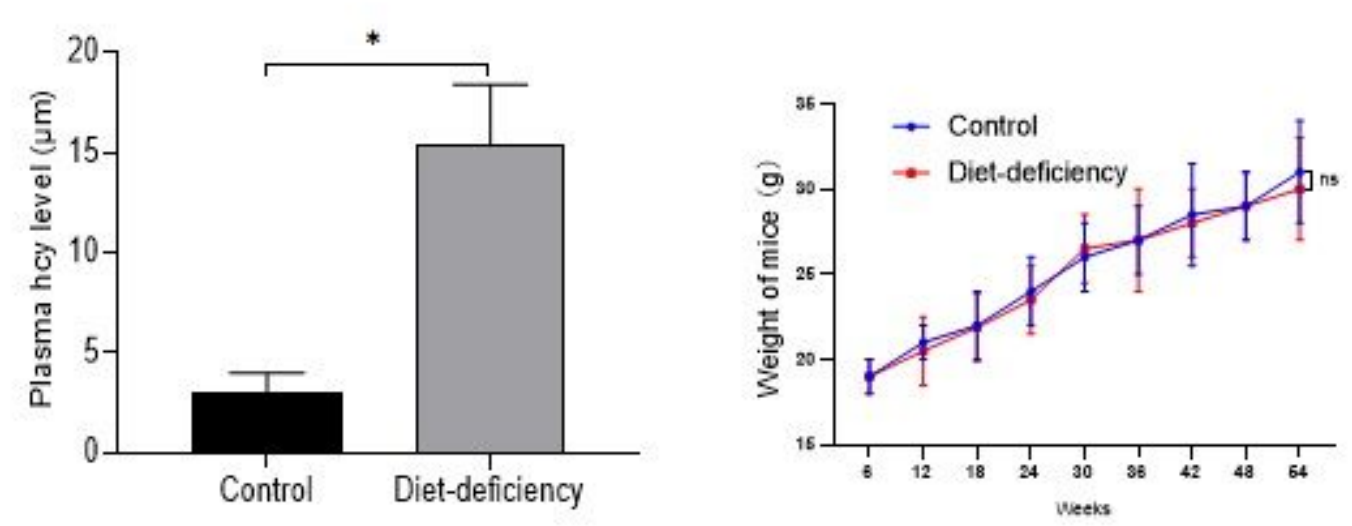

$\mathrm{C}$

D
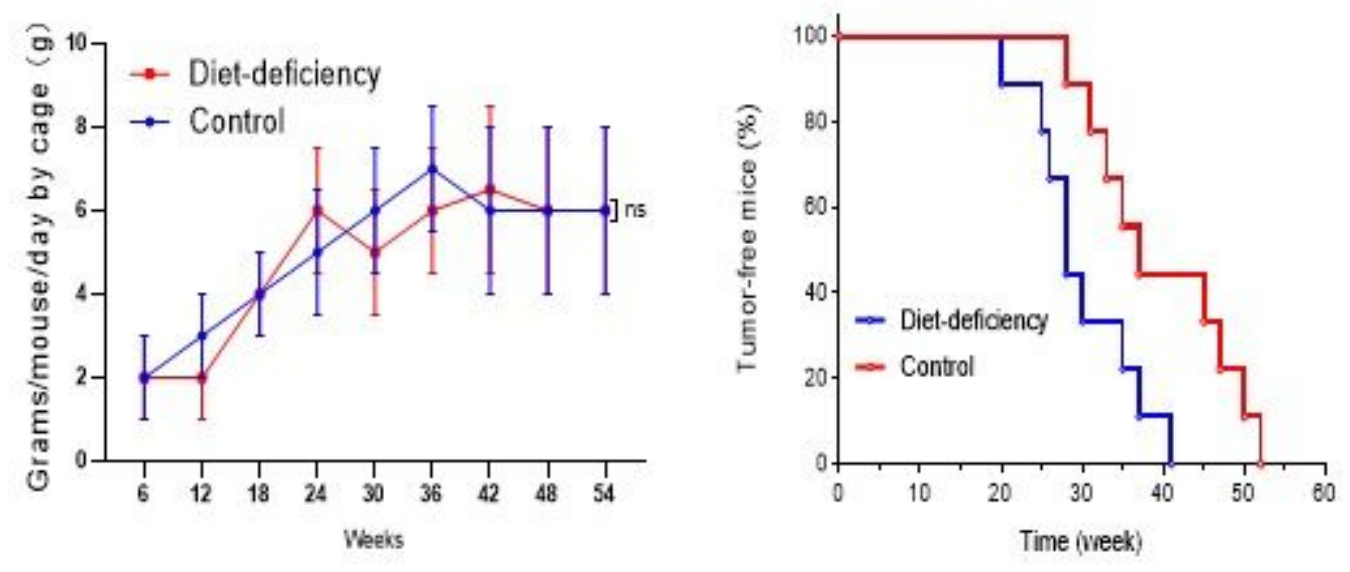

Figure 1

FVD diet induces HHcy and reduces tumor latency in MMTV-ErbB2 mice. (A) MMTV-ErbB2 mice fed for 4 weeks with a FVD diet had higher level of plasma homocysteine compared with mice fed with vehicle diet. No significant differences were observed in (B) body weight and (C) food consumption between MMTV-ErbB2 mice fed with a FVD diet or control. (D) The FVD diet increases mammary tumor latency in MMTV-ErbB2 mice. Values are presented the mean \pm SD. ${ }^{*} P<0.05$. FVD, Folate and vitamin B12 deficient; MMTV, mouse mammary tumor virus; ErbB2, erb-b2 receptor tyrosine kinase 2; HHcy, hyperhomocysteinemia. 


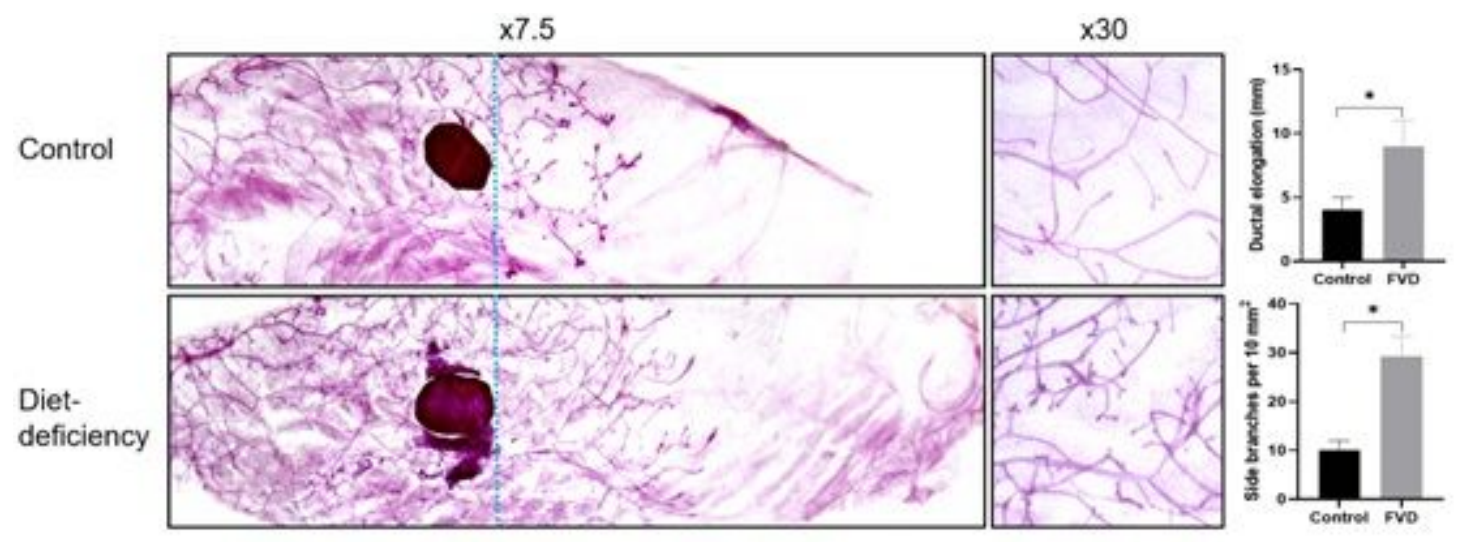

\section{Figure 2}

Mammary tissues were collected from 18-week-old control and FVD diet-fed MMTV-ErbB2 mice for whole mount analyses. Representative images of mammary whole mounts from MMTV-ErbB2 mice fed with a FVD diet or control are shown at increasing magnifications (7.5x, and 30x magnifications). The FVD diet alters mammary morphogenesis in MMTV-ErbB2 mice. The FVD diet increased ductal extension beyond the lymph node and side branches in the mammary whole mounts. FVD, Folate and vitamin B12 deficient; MMTV, mouse mammary tumor virus; ErbB2, erb-b2 receptor tyrosine kinase.

HE

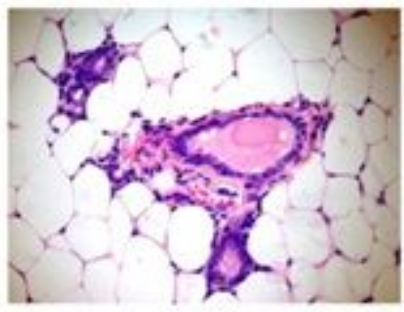

Control

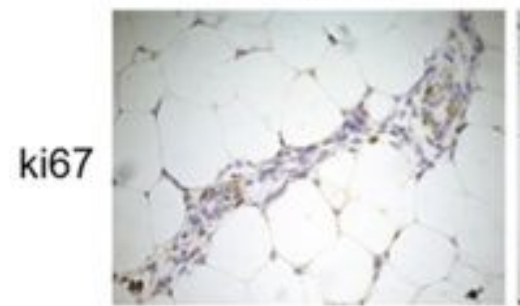

Control

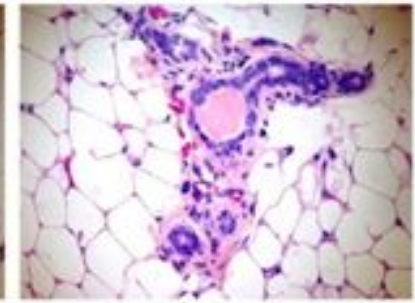

Diet-deficiency

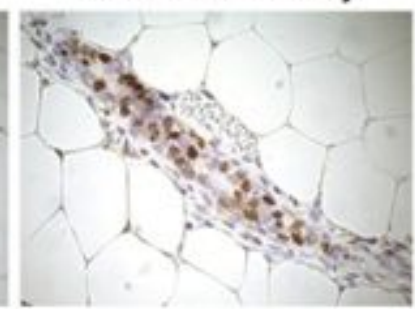

Diet-deficiency

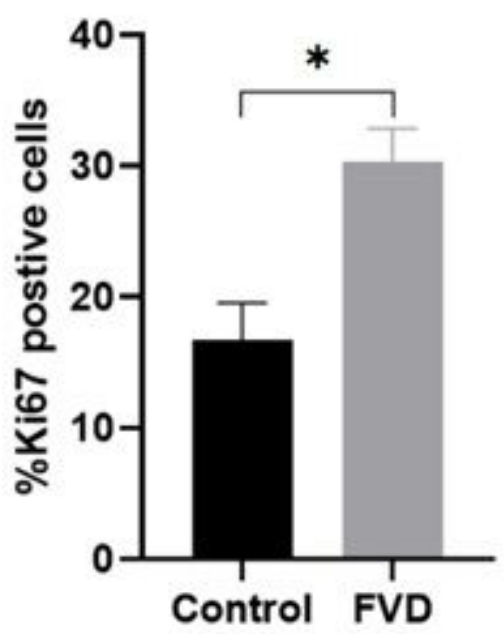

\section{Figure 3}

Hematoxylin and eosin-stained tumor sections and Ki67 positive cells in mammary glands of FVD or control diet-fed MMTV-ErbB2 mice. The FVD diet promoted mammary morphogenesis and increased Ki67 positive cells in MMTV-ErbB2 mice. Data are presented as the mean $\pm \mathrm{SEM}$. ${ }^{*} \mathrm{P}<0.01$ vs. control. FVD, Folate and vitamin B12 deficient; MMTV, mouse mammary tumor virus; ErbB2, erb-b2 receptor tyrosine kinase 2. 
A

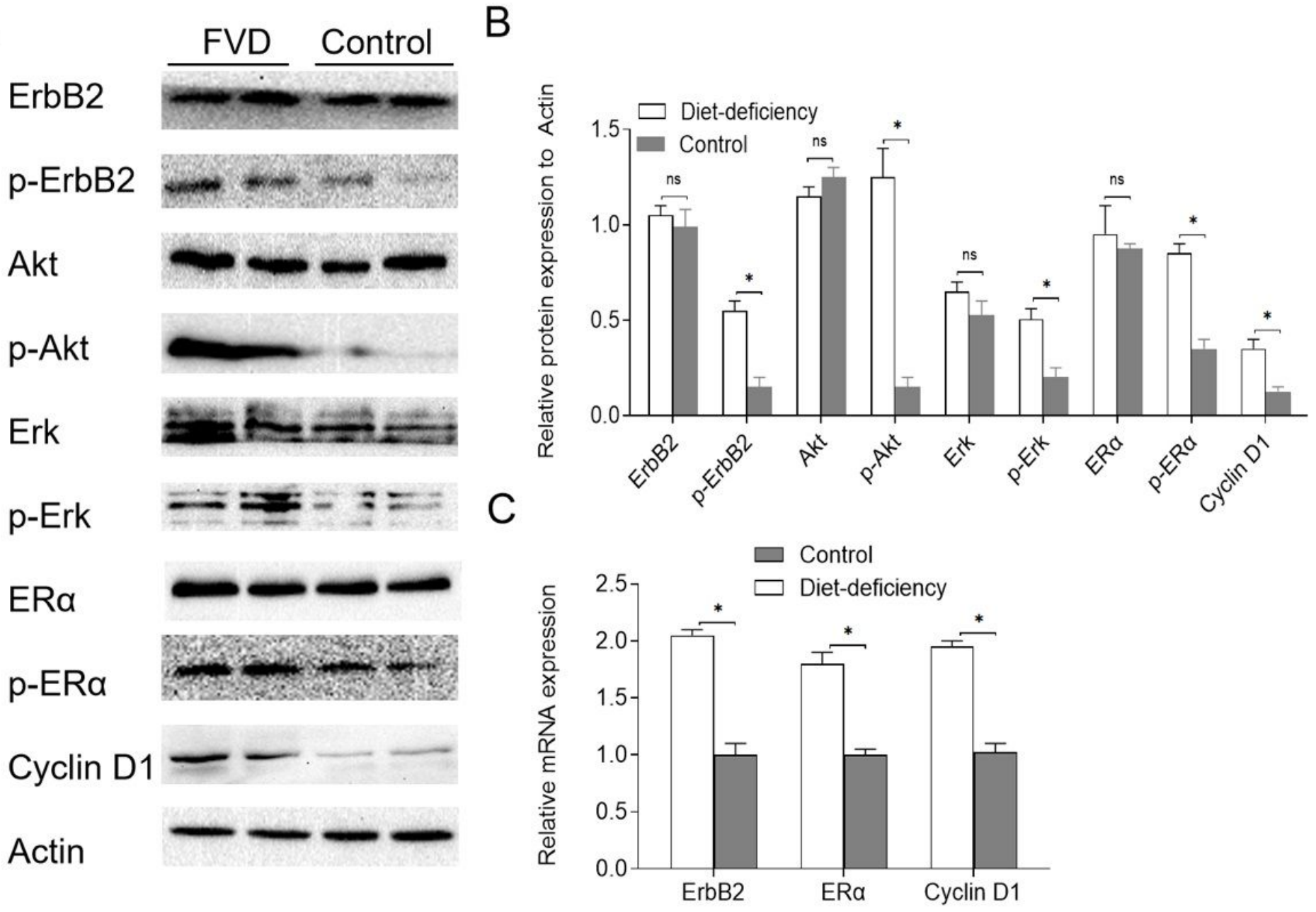

Figure 4

FVD diet enhances the activation of RTK and ERa signaling in premalignant mammary tissues from MMTV-ErbB2 mice. Protein and RNA lysates were collected from the mammary glands of 18-week-old control and FVD-treated mice ( $\mathrm{n}=3$ mice/group). ( $\mathrm{A}$ and $\mathrm{B}$ ) Expression levels of total and phosphorylated protein markers of RTK and ERa pathways were detected using western blotting. (C) The relative mRNA levels for the indicated genes were measured using reverse transcription-quantitative PCR. Data are presented as the mean $\pm \mathrm{SEM}$. ${ }^{*} \mathrm{P}<0.01$ vs. control. FVD, Folate and vitamin B12 deficient; MMTV, mouse mammary tumor virus; ErbB2, erb-b2 receptor tyrosine kinase 2; ER, estrogen receptor; RTK, receptor tyrosine kinase. 